\title{
Predicting the mechanical characteristics of hydrogen functionalized graphene sheets using artificial neural network approach
}

\author{
Venkatesh Vijayaraghavan ${ }^{1 *}$, Akhil Garg ${ }^{1}$, Chee How Wong ${ }^{1}$, Kang Tai ${ }^{1}$ and Yogesh Bhalerao ${ }^{2}$
}

\begin{abstract}
The mechanical properties of hydrogen functionalized graphene (HFG) sheets were predicted in this work by using artificial neural network approach. The predictions of tensile strength of HFG sheets made by the proposed approach are compared to those generated by molecular dynamics simulations. The results indicate that our proposed computing technique can be used as a powerful tool for predicting the tensile strength of the HFG sheet.
\end{abstract}

Keywords: Hydrogen functionalized graphene; Tensile; Atomistic simulation; Nanomechanics; Artificial neural network

\section{Background}

Research in graphene has attracted significant interest in recent years due to its remarkable mechanical [1] and physical properties [2,3]. A single-layer graphene sheet has the thickness of only one carbon atom which makes it the thinnest material [4] with a large specific surface area [5]. This feature of graphene makes it an ideal candidate for nanoelectromechanical systems (NEMS) [6] and nanofluidic devices. These future applications require a critical understanding of the exceptional mechanical properties of graphene for its application in NEMS and nanolevel biological devices. Theoretical studies on graphene are a popular mode of research, employing $a b$ initio calculations or molecular dynamics (MD) simulation technique.

Application of soft computing methods such as artificial neural networks (ANN), genetic programming, and fuzzy logic can be used as an alternative method for modeling complex behavior of materials such as graphene. These methods require input training data which can be obtained from the analytical tools such as MD that is based on a specific geometry and temperature. Based on the input, the proposed computing method can then be able to generate meaningful solutions for complicated problems [7-11]. Additionally, among the

\footnotetext{
* Correspondence: ve0005an@e.ntu.edu.sg

'School of Mechanical and Aerospace Engineering, Nanyang Technological

University, 50 Nanyang Avenue, Singapore 639798, Singapore

Full list of author information is available at the end of the article
}

various soft computing methods described above, ANN offers the advantage of a fast and cost-effective formulation of a mathematical model based on multiple variables with no existing analytical models [12,13]. It is to the best of author's knowledge that limited or no work exists on the application of soft computing models on the tensile properties of graphene sheets.

Hence, in the present work, we have proposed ANN method to model the elastic characteristics of hydrogen functionalized graphene (HFG). The values of tensile strength of HFG generated by MD simulations are further fed into the paradigm of ANN.

\section{Nanoscale material modeling by MD simulation}

The classical MD method is deployed to carry out the numerical simulation. In this capacity, Newton's equations of motion are computed by means of Brenner's secondgeneration bond order (REBO) function [14] for the set of atoms which are covalently bonded. The REBO potential is able to accurately describe the properties of solid-state and molecular carbon nanostructures $[15,16]$ while maintaining the accuracies of the $a b$ initio and semi-empirical methods in simulating large systems [15-21]. The mathematical form of the potential equations is defined as

$$
E_{\mathrm{REBO}}=V_{\mathrm{R}}\left(r_{i j}\right)-b_{i j} V_{\mathrm{A}}\left(r_{i j}\right),
$$

where $V_{\mathrm{R}}\left(r_{i j}\right)$ and $V_{\mathrm{A}}\left(r_{i j}\right)$ are the repulsive and attractive pair terms and $b_{i j}$ term is used to include the reactive empirical bond order between the atoms. 


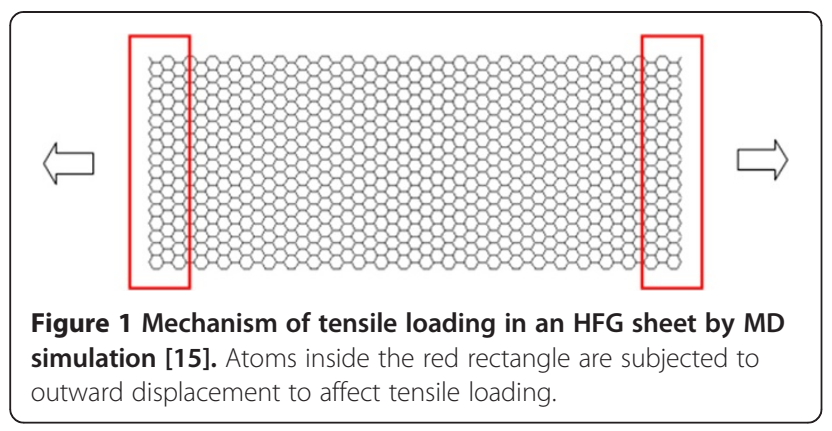

The MD simulation procedure described here is similar to our previous work as can be seen in [15]. The tensile loading procedure of HFG sheet is shown in Figure 1. As an illustration, the morphological characteristics of the HFG sheet of length $100 \AA$ under tension is depicted in Figure 2 . The data hence generated by our MD simulation agrees very well with the literature $[15,20]$, and hence, we can validate the reliability of our MD simulation.

\section{Methods}

The data obtained by the MD simulation are shown in Tables 1 and 2 which represent the data for training and testing samples, respectively. The main inputs considered in our study are the percentage of hydrogen functionalization and simulation temperature. The output of our study is the tensile strength of HFG. In order to explicitly test the extrapolation capability of method, 20
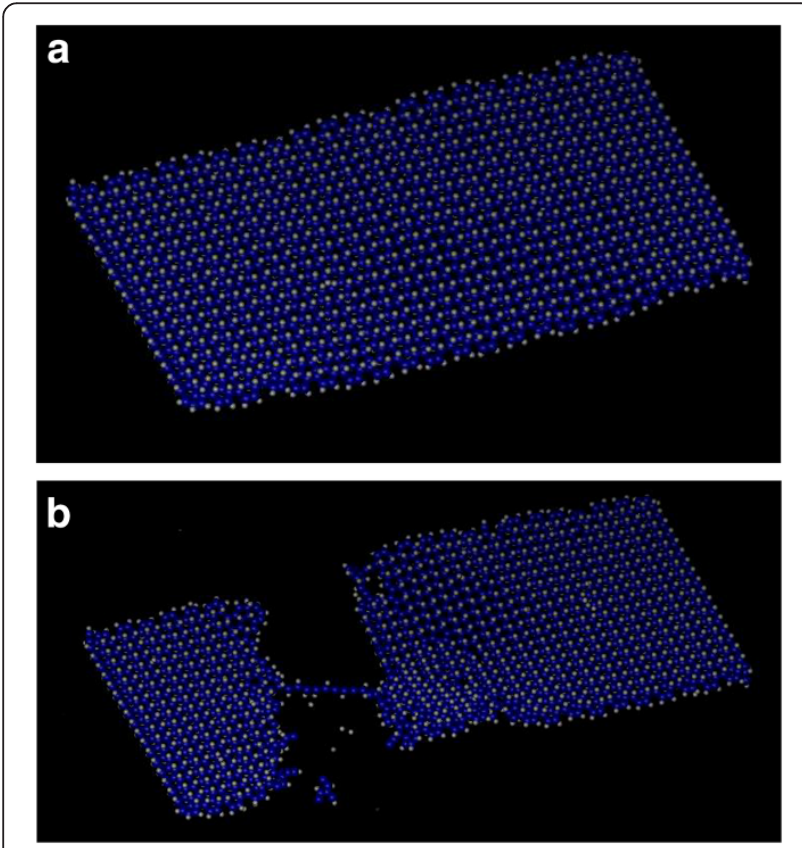

Figure 2 Tensile loading applied on an HFG sheet [15]. The graphene sheet maintains its stable shape (a) when no load is applied, further application of tensile load results in breaking and fragmentation as shown in (b).
Table 1 Data for training obtained by MD simulation

\begin{tabular}{|c|c|c|}
\hline Temperature (K) & $\begin{array}{l}\text { Percentage of hydrogen } \\
\text { functionalization }\end{array}$ & $\begin{array}{c}\text { Tensile } \\
\text { strength (GPa) }\end{array}$ \\
\hline 0 & 0 & 157 \\
\hline 0 & 0.1 & 135 \\
\hline 0 & 0.2 & 123 \\
\hline 0 & 0.3 & 109 \\
\hline 0 & 0.4 & 92 \\
\hline 0 & 0.5 & 85 \\
\hline 0 & 0.6 & 82 \\
\hline 0 & 0.7 & 83 \\
\hline 0 & 0.8 & 85 \\
\hline 0 & 0.9 & 81 \\
\hline 0 & 1 & 87 \\
\hline 300 & 0 & 101 \\
\hline 300 & 0.1 & 72 \\
\hline 300 & 0.2 & 63 \\
\hline 300 & 0.3 & 52 \\
\hline 300 & 0.4 & 43 \\
\hline 300 & 0.5 & 39 \\
\hline 300 & 0.6 & 34 \\
\hline 300 & 0.7 & 35 \\
\hline 300 & 0.8 & 35 \\
\hline 300 & 0.9 & 41 \\
\hline 300 & 1 & 43 \\
\hline
\end{tabular}

samples are used for training and 11 are used for testing. Training samples consist of values of tensile strength at temperatures of 0 and $300 \mathrm{~K}$, whereas testing samples consist of values of temperature at $600 \mathrm{~K}$.

\section{Nanoscale material modeling by ANN approach}

In this paper, we proposed ANN approach. In this approach, data obtained from MD is fed into the paradigm

\section{Table 2 Data for testing obtained by MD simulation}

\begin{tabular}{ccc}
\hline Temperature (K) & $\begin{array}{c}\text { Percentage of hydrogen } \\
\text { functionalization }\end{array}$ & $\begin{array}{c}\text { Tensile } \\
\text { strength (GPa) }\end{array}$ \\
\hline 600 & 0 & 51 \\
600 & 0.1 & 35 \\
600 & 0.2 & 32 \\
600 & 0.3 & 25 \\
600 & 0.4 & 20 \\
600 & 0.5 & 19 \\
600 & 0.6 & 15 \\
600 & 0.7 & 18 \\
600 & 0.8 & 18 \\
600 & 0.9 & 21 \\
600 & 1 & 22 \\
\hline
\end{tabular}




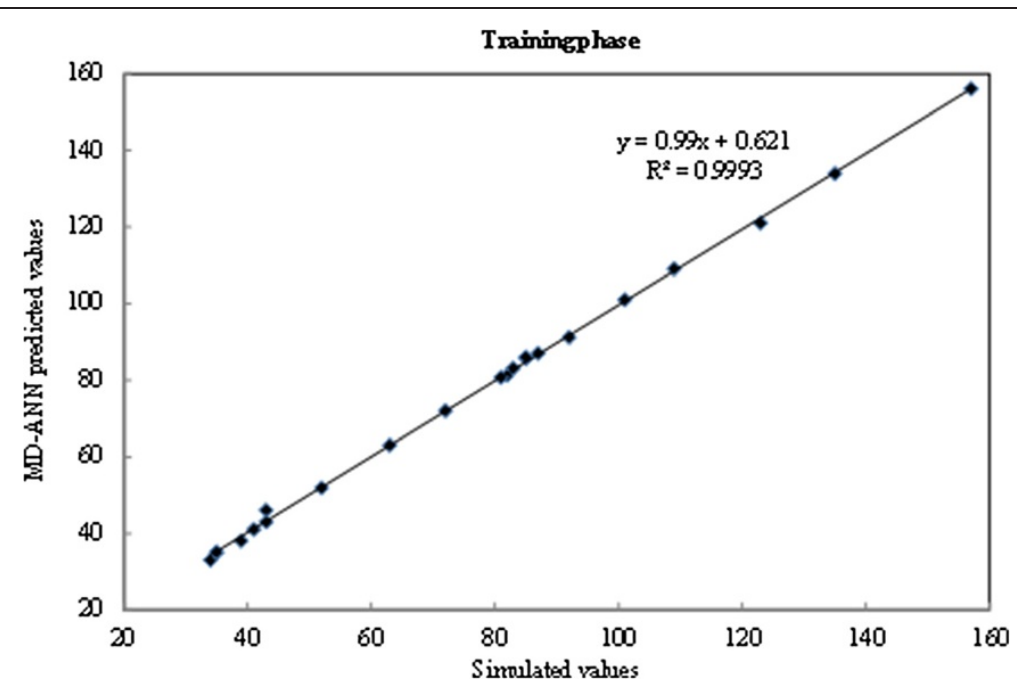

Figure 3 Comparison between predicted values and simulated values on training data.

of ANN for training of network. ANN network consist of three layers: input, hidden, and output layers. Input layer consist of two neurons since there are two inputs [22-31]. The number of neurons in the hidden layer is chosen based on trial-and-error method. The output layer comprise of single neuron, i.e., output of the system. The neurons of one layer to the neurons of preand-after layer are connected through weighted links.

Weights are initialized and are multiplied by input values specified by each neuron. The neuron estimate summation of weighted inputs and passes it to the transfer function $(A)$ which produces an output $Y_{p}$.

$$
Y_{p}=A\left(\sum_{i=0}^{n-1} w_{i} x_{i}-\delta\right)
$$

where $w_{i}$ is weight, $x_{i}$ is the $i$ th input variable, and $\delta$ is the threshold or offset of the neuron. The activation function used is sigmoid logistic function given by

$$
A(x)=\frac{1}{1+e^{-x}}
$$

The difference between the output value of network and actual value for a sample $i$ is given by

$$
\text { Error }_{m}=\frac{1}{2} \sum_{m=1}^{M}\left(A c_{i}-M_{i}\right)^{2}
$$

where $A c_{i}$ and $M_{i}$ are actual and predicted values for $i$ th sample, respectively, and $M$ is the number of neurons in

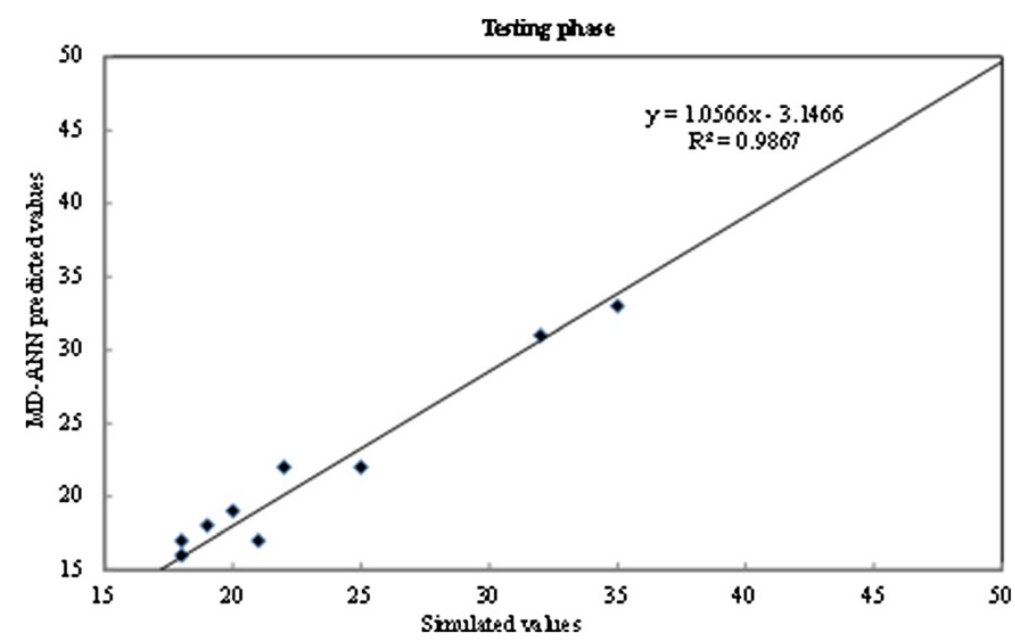

Figure 4 Comparison between predicted values and simulated values on testing data. 
the output of network. The average error for the whole network is given by

$$
\text { Error }_{m}=\frac{1}{2} \sum_{m=1}^{M} \sum_{m=1}^{M}\left(A c_{i}-M_{i}\right)^{2},
$$

where $N$ is the total number of samples. The LevenbergMarquardt algorithm [32] that works on the principle of the second derivative is used to optimize the $\operatorname{Error}_{p}$. The simpler form of Hessian matrix is used and the algorithm iterates weights using formulae

$$
x_{k+1}=x_{k}-\left[J^{T} J+\mu I\right]^{-1} J^{T} e,
$$

where $j$ is the Jacobian matrix that consists of the first derivatives of the network errors, $e$ is a vector of network errors, $\mu$ is the learning rate, and $I$ is the identity matrix. The weights are updated by LMA until the threshold error is achieved. The computation of weights is iterative and it consumes time.

In the present work, feed-forward network of three layers is implemented in MATLAB R2010b. The number of neurons in hidden layer is determined based on the minimum value of root-mean-square error (RMSE) of the model on the training data set. A trial-and-error approach is adopted to select the number of neurons in the hidden layers. It was found that for number of neurons six, the RMSE is minimum, and therefore, the ANN model with single hidden layer of six neurons is selected. The performance of the selected ANN model is discussed in the 'Performance comparison of proposed approach' section.

\section{Results and discussion}

The predictions obtained from the proposed approach is compared to those generated using MD simulations based on square of correlation coefficient $\left(R^{2}\right)$ given by

$$
R^{2}=\left(\frac{\sum_{i=1}^{n}\left(A_{i}-\bar{A}_{i}\right)\left(M_{i}-\bar{M}_{i}\right)}{\sqrt{\sum_{i=1}^{n}\left(A_{i}-\bar{A}_{i}\right)^{2} \sum_{i=1}^{n}\left(M_{i}-\bar{M}_{i}\right)^{2}}}\right),
$$

where $M_{i}$ and $A_{i}$ are predicted and actual values, respectively, $\bar{M}_{i}$ and $\bar{A}_{i}$ are the average values of predicted and actual, respectively, and $n$ is number of training samples.

The results obtained from the simulation studies and predicted by using proposed approach on training and testing data is shown in Figures 3 and 4, respectively. The graph shown in Figure 3 indicates that the proposed approach has impressively well learned the non-linear relationship between the input and output process parameters with high correlation values. The result of testing phase shown in Figure 4 indicates that the values predicted by MD-ANN approach are well in agreement with the simulated values.

\section{Conclusion}

The performance of the proposed MD-ANN approach is evaluated in the 'Performance comparison of proposed approach' section. The results conclude that the MDANN model have shown excellent generalization ability with high statistical values of $R^{2}$ on testing data. The high generalization ability of the MD-ANN model is beneficial for MD experts who are currently looking for high fidelity models that predict the tensile strength of graphene under uncertain input process conditions, and therefore, the cost of having to run additional MD simulations can be avoided. The model can be used offline for prediction and can be further optimized to determine the optimum input process parameters that maximize the tensile strength of nanomaterials.

\section{Competing interests}

The authors declare that they have no competing interests.

\section{Authors' contributions}

W carried out preliminary simulations to confirm that data generated confirm to literature and is supervised by CHW. AG carried out the computational simulations designed and formulated by $Y B$ and is supervised by KT. All authors read and approved the final manuscript.

\section{Acknowledgements}

W and AG gratefully acknowledge the financial support in form of scholarship provided by the School of Mechanical and Aerospace Engineering, Nanyang Technological University, Singapore.

\section{Author details}

${ }^{1}$ School of Mechanical and Aerospace Engineering, Nanyang Technological University, 50 Nanyang Avenue, Singapore 639798, Singapore. ${ }^{2}$ MIT Academy of Engineering (MAE), Pune, Maharashtra, India.

Received: 30 September 2013 Accepted: 23 October 2013 Published: 13 Nov 2013

\section{References}

1. Lee, C, Wei, X, Kysar, JW, Hone, J: Measurement of the elastic properties and intrinsic strength of monolayer graphene. Science 321(5887), 385-388 (2008)

2. Balandin, AA, Ghosh, S, Bao, WZ, Calizo, I, Teweldebrhan, D, Miao, F, Lau, $\mathrm{CN}$ : Superior thermal conductivity of single-layer graphene. Nano Lett 8(3), 902-907 (2008)

3. Williams, JR, DiCarlo, L, Marcus, CM: Quantum hall effect in a gate-controlled p-n junction of graphene. Science 317(5838), 638-641 (2007)

4. Novoselov, KS, Geim, AK, Morozov, SV, Jiang, D, Zhang, Y, Dubonos, SV, Grigorieva, IV, Firsov, AA: Electric field effect in atomically thin carbon films. Science 306(5696), 666-669 (2004)

5. Geim, AK: Graphene: status and prospects. Science 324(5934), 1530-1534 (2009)

6. Standley, B, Bao, WZ, Zhang, H, Bruck, J, Lau, CN, Bockrath, M: Graphene-based atomic-scale switches. Nano Lett 8(10), 3345-3349 (2008)

7. Kordon, A, Castillo, F, Smits, G, Kotanchek, M: Application issues of genetic programming in industry. Gen Prog Theory Prac III 9, 241-258 (2006)

8. Reihanian, M, Asadullahpour, SR, Hajarpour, S, Gheisari, KH: Application of neural network and genetic algorithm to powder metallurgy of pure iron. Mat Design 32(6), 3183-3188 (2011)

9. Ramanan, TR, Sridharan, R, Shashikant, KS, Haq, AN: An artificial neural network based heuristic for flow shop scheduling problems. J Intell Manufactur 22(2), 279-288 (2011)

10. Liao, KW, Fan, JC, Huang, CL: An artificial neural network for groutability prediction of permeation grouting with microfine cement grouts. Comp Geotechnics 38(8), 978-986 (2011)

11. Garg, A, Tai, K: Comparison of statistical and machine learning methods in modelling of data with multicollinearity. Int J Modell Identification Control 18(4), 295-312 (2013) 
12. Garcia-Mateo, C, Capdevila, C, Caballero, FG, de Andres, CG: Artificial neural network modeling for the prediction of critical transformation temperatures in steels. J Mater Sci 42(14), 5391-5397 (2007)

13. Garg, A, Tai, K, Lee, CH, Savalani, MM: A hybrid M5'-genetic programming approach for ensuring greater trustworthiness of prediction ability in modelling of FDM process. J Intell Manufactur doi: 10.1007/s10845-013-0734-1

14. Brenner, DW, Shenderova, OA, Harrison, JA, Stuart, SJ, Ni, B, Sinnott, SB: A second-generation reactive empirical bond order (REBO) potential energy expression for hydrocarbons. J Physics-Condensed Matter 14(4), 783-802 (2002)

15. Wong, $\mathrm{CH}$, Vijayaraghavan, $\mathrm{V}$ : Nanomechanics of free form and water submerged single layer graphene sheet under axial tension by using molecular dynamics simulation. Mater Sci Engineer A 556, 420-428 (2012)

16. Wong, $\mathrm{CH}$, Vijayaraghavan, $\mathrm{V}$ : Nanomechanics of imperfectly straight single walled carbon nanotubes under axial compression by using molecular dynamics simulation. Comp Mater Sci 53(1), 268-277 (2012)

17. Vijayaraghavan, $\mathrm{V}$, Wong, CH: Nanomechanics of single walled carbon nanotube with water interactions under axial tension by using molecular dynamics simulation. Comp Mater Sci 79, 519 (2013)

18. Vijayaraghavan, $\mathrm{V}$, Wong, CH: Shear deformation characteristics of single walled carbon nanotube with water interactions by using molecular dynamics simulation. Physica E Low-Dimensional Sys Nanostructures doi:/ 10.1016/j.physe.2013.06.025

19. Vijayaraghavan, $\mathrm{V}$, Wong, $\mathrm{CH}$ : Temperature, defect and size effect on the elastic properties of imperfectly straight carbon nanotubes by using molecular dynamics simulation. Comp Mater Sci 71, 184-191 (2013)

20. Vijayaraghavan, $\mathrm{V}$, Wong, $\mathrm{CH}$ : Tensile loading characteristics of free-form and water submerged single layer graphene sheet. Proc 12th IEEE Confer Nanotechnol (2012). doi: 10.1109/NANO.2012.6321916

21. Wong, $\mathrm{CH}$, Vijayaraghavan, $\mathrm{V}$ : Nanomechanics of nonideal single- and doublewalled carbon nanotubes. J Nanomaterials doi: 10.1155/2012/490872

22. Garg, A, Rachmawati, L, Tai, K: Classification-driven model selection approach of genetic programming in modelling of turning process. Int J Advanced Manufactur Technol doi: 10.1007/s00170-013-5103-x

23. Garg, A, Savalani, MM, Tai, K: State-of-the-Art in empirical modeling of rapid prototyping processes. Rapid Prototyping $J$ in press

24. Garg, A, Tai, K: A hybrid genetic programming-artificial neural network approach for modeling of vibratory finishing process. Int Proc Comp Sci Info Technol (IPCSIT) 18, 14-19 (2011)

25. Garg, A, Tai, K: Comparison of regression analysis, artificial neural network and genetic programming in handling the multicollinearity problem, pp. 353-358. Proceedings of 2012 International Conference on Modelling, Identification and Control (ICMIC 2012), Wuhan, China (2012)

26. Benyelloul, K, Aourag, H: Elastic constants of austenitic stainless steel: Investigation by the first-principles calculations and the artificial neural network approach. Comp Mater Sci 67, 353-358 (2013)

27. Garg, A, Sriram, S, Tai, K: Empirical Analysis of Model Selection Criteria for Genetic Programming in Modeling of Time Series System, pp. 84-88. Proceedings of 2013 IEEE Conference on Computational Intelligence for Financial Engineering \& Economics (CIFEr), Singapore (2013)

28. Garg, A, Tai, K: Selection of a Robust Experimental Design for the Effective Modeling of Nonlinear Systems using Genetic Programming, pp. 293-298. Proceedings of 2013 IEEE Symposium Series on Computational Intelligence and Data mining (CIDM), Singapore (2013)

29. Garg, A, Bhalerao, Y, Tai, K: Review of empirical modeling techniques for modeling of turning process. Int J Modell Identification Control 20(2), 121-129 (2013)

30. Esmailzadeh, M, Aghaie-Khafri, M: Finite element and artificial neural network analysis of ECAP. Comp Mater Sci 63, 127-133 (2012).

31. Garg, A, Vijayaraghavan, V, Mahapatra, SS, Tai, K, Wong, CH: Performance evaluation of microbial fuel cell by artificial intelligence methods. Expert systems with applications (2013). doi: 10.1016/j.eswa.2013.08.038

32. Cherkassky, V, Ma, Y: Comparison of model selection for regression. Neural Comput 15(7), 1691-1714 (2003)

10.1186/2193-8865-3-83

Cite this article as: Vijayaraghavan et al:: Predicting the mechanical characteristics of hydrogen functionalized graphene sheets using artificial neural network approach. Journal Of Nanostructure in Chemistry 2013, 3:83 